\title{
Infrared surface plasmon spectroscopy and biosensing
}

\author{
Victor Yashunsky*a, Alexander Zilbershtein ${ }^{\mathrm{a}}$, Vladislav Lirtsman ${ }^{\mathrm{a}}$, Tal Marciano ${ }^{\mathrm{a}}$, \\ Benjamin Aroeti ${ }^{\mathrm{b}}$, Michael Golosovsky ${ }^{\mathrm{a}}$, Dan Davidov ${ }^{\mathrm{a}}$ \\ ${ }^{a}$ The Racah Institute of Physics, the Hebrew University of Jerusalem, Jerusalem, Israel, 91904; \\ ${ }^{b}$ Department of Cell and Developmental Biology, the Alexander Silberman Institute of Life Science, \\ the Hebrew University of Jerusalem, Jerusalem, Israel, 91904
}

\begin{abstract}
The cell morphology is a valuable indicator of the physical condition and general status of the cell. Here we demonstrate a methodology for noninvasive biosensing of adherent living cells. Our method is based on infrared reflection spectroscopy of living cells cultured on thin Au film. To characterize cell morphology we utilized the unique properties of the infrared surface plasmon $(\lambda=1-3 \mu \mathrm{m})$ and infrared guided wave that travel inside the cell monolayer. We demonstrate that our method enables monitoring of submicron variations in cell morphology in real-time and in a labelfree manner. In addition to morphological characterization, our method allows investigation of chemical composition and molecular structure of cells through infrared absorption spectroscopy analysis.
\end{abstract}

Keywords: Living Cells, Biosensor, Surface Plasmon, Label-free, Noninvasive method, Evanescent Waves, Infrared spectroscopy

\section{INTRODUCTION}

Our group has recently developed a label-free biosensing method based on the infrared (IR) Surface Plasmon (SP) spectroscopy ${ }^{1-4}$. This method enabled us to study diverse dynamic properties of large bio-entities in water-based medium, such as living cells. We exploited infrared surface plasmon (SP) and waveguide (TM) modes to study adherent cells and cell layers. The surface plasmon wave propagates at the cell/substrate interface and probes the lower (basal) part of the cell ${ }^{3}$ while the waveguide modes travel inside the cell monolayer and are sensitive to cell height and cell-cell adhesion. Simultaneous analysis of the mode wavelength and attenuation provide information on submicron morphological changes in living cells and enables infrared absorption spectroscopy of living cells.

* victor.yashunsky@mail.huji.ac.il; phone 972-2-6586-338; fax 972-2-5617-805; 


\section{METHODOLOGY}

\subsection{Surface plasmon in biosensing}

The surface plasmon (SP) is a transverse magnetic (TM) wave that travels along the metal - dielectric interface (xdirection) and decays in the direction perpendicular to the interface (z-direction) ${ }^{5}$. SP can be resonantly excited with light incident on the metal-dielectric interface using special couplers, such as prism (Otto, Kretschmann configurations) or grating. The surface plasmon resonance appears as a sharp dip in the angular/wavelength-dependent reflectivity. One can follow the change of resonant angle/wavelength to detect delicate changes in the dielectric (analyte). For wavelength interrogation in Kretschmann configuration we can write inexplicit expression for the SP resonant wavelength:

$$
n\left(\lambda_{S P}\right)=n_{p} \sin \theta\left(\frac{\left|\varepsilon_{m}\right|}{\left|\varepsilon_{m}\right|+n_{p}^{2} \sin ^{2} \theta_{i n c}}\right)^{1 / 2}
$$

Here, $n$ and $n_{p}$ are the refractive indices of the analyte and the prism correspondingly, $\varepsilon_{m}$ is the dielectric permittivity of the metal and $\theta_{\text {inc }}$ is the angle of incidence [Fig. 1(a)]. Thus, one can follow changes in $n$ by measuring the shift of the SP resonant wavelength, $\lambda_{S P}$. The confinement of the SP wave in z-direction makes it a highly sensitive tool for detection of small changes in refractive index $\left(10^{-7} \mathrm{RIU} \text { ) that occur close to the metal surface (at a distance of } \sim 0.15 \mu \mathrm{m}\right)^{1,5,6}$. This principle was successfully applied in variety of biosensors, but mainly for detection of molecular binding ${ }^{7}$. For efficient sensing of larger objects, like living cells, the SP sampling depth should be extended. For infrared wavelength the SP sampling depth achieves several microns $(2 \mu \mathrm{m}$ at $\lambda=2.5 \mu \mathrm{m})$. This makes possible to probe significant part of cell interior.

In the context of cell biosensing, measurement of SP wavelength reveals the refractive index $n$ which is proportional to the total cell volume inside the SP field. Measurement of SP resonance depth (Q-factor) reveals the lossy part of refractive index, $\kappa$. The latter is determined by scattering and absorption of the SP wave. Structural changes taking place in living cells contribute mostly to the scattering term ${ }^{3}$ when chemical composition and molecular structure of the cells determines the absorption.

\section{EXPERIMENTAL SETUP}

\subsection{Experimental setup for infrared surface plasmon excitation}

Our experimental setup is schematically shown in Fig. 1(a). The Au-coated right-angle $\mathrm{ZnS}$ prism [20x40 $\mathrm{mm}^{2}$ base (ISP Optics, Inc., Irvington, N.Y, US)] is attached to a $1.5 \mathrm{ml}$ flow chamber equipped with temperature-stabilizer. The cell medium flow in the chamber is controlled by the motorized bee syringe pump equipped with a variable speed controller. The live cells were directly cultured on top of the Au-coated prism surface unless otherwise stated. The prism-flow chamber assembly was mounted on the vertical translation stage to allow measurements at different incident angles, $\theta_{\text {inc }}$. 
(a)

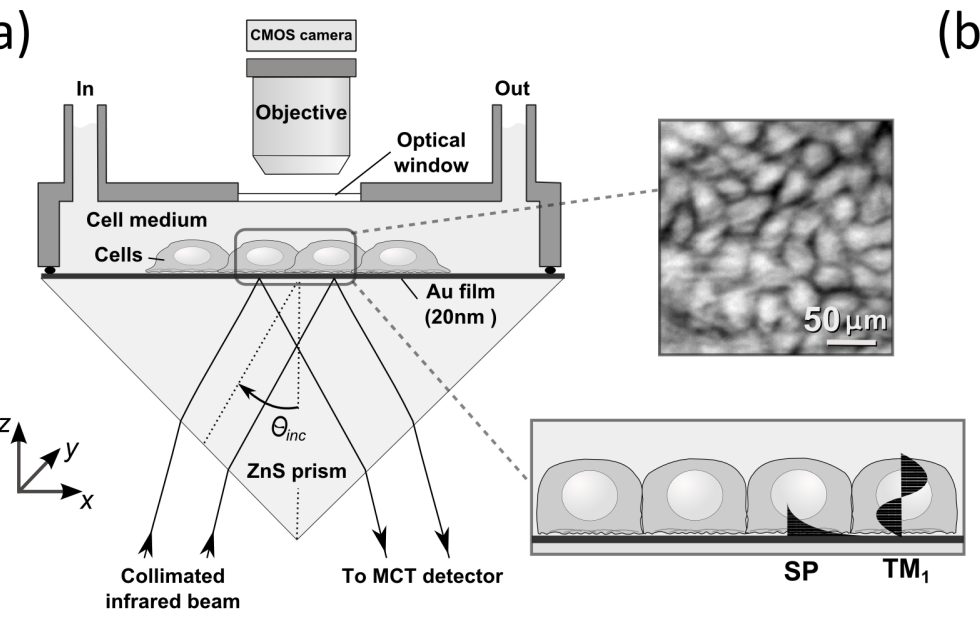

(b)

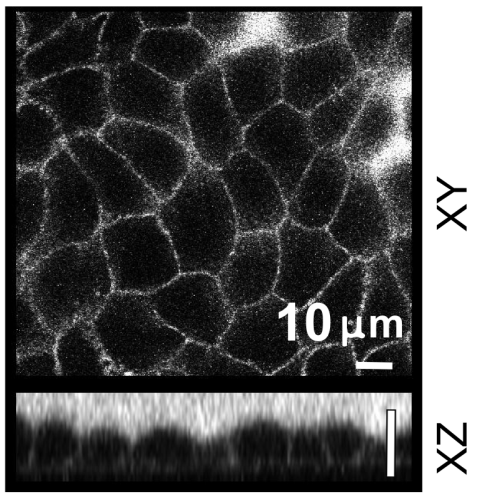

Figure 1. (a) Experimental setup for SP spectroscopy of living cell layers. The collimated infrared beam impinges on the Au-coated prism attached to flow chamber. In addition to SP mode that probes lower cell portion interconnected cells enable excitation waveguide modes (TM) that is sensitive to whole cell body. By measuring the intensity of the reflected beam one can monitor cellular processes. The cells on top of the Au-coated prism imaged through the optical window. (b) Confocal microscopy image of a MDCK cell monolayer immersed in growth medium containing $0.1 \mu \mathrm{M}$ sulforhodamine green (SRG). The SRG penetrates between the cells and allows to see the cell boundaries while the cells appear as dark objects. The XZ image was reconstructed from series of $\mathrm{XY}$ images taken with $0.5 \mu \mathrm{m}$ step along the z-axis. The vertical bar indicates $10 \mu \mathrm{m}$.

\subsection{Cell maintenance}

The Madin-Darby canine kidney cells (MDCK) were routinely cultured in the minimal essential medium (MEM, Biological Industries, Beit Haemek, Israel), supplemented with 5\% (vol/vol) fetal calf serum (FCS) and 1\% (vol/vol) antibiotics (Biological Industries). During the measurements the cells were bathed in fresh MEM which was constantly replaced at slow flow $(5-50 \mu \mathrm{l} / \mathrm{min})$.

\subsection{Measurement Protocol}

The reflection infrared spectroscopy was performed using Kretschmann's geometry. The IR beam emitted from the external port of the FTIR spectrometer [Bruker IFS 55 Equinox FTIR] equipped with a tungsten lamp and $\mathrm{KBr}$ beam splitter, was collimated, polarized, reflected from the prism and focused onto a liquid-nitrogen-cooled MCT (HgCdTe) detector. Each spectrum lasted $\sim 25 \mathrm{sec}$ and represented an average of 8 scans $\left(2-8 \mathrm{~cm}^{-1}\right.$ resolution). The $s$-polarized spectrum was used as a background. In case of kinetic measurements the $s$-polarized spectrum measured at $\mathrm{t}=0$ was used as a background for all subsequent spectra.

\section{RESULTS}

\subsection{Monitoring of living cells by surface plasmon spectroscopy}

We focus here on surface plasmon sensing of living cells cultured on top of the Au-coated substrate. The cells were immersed in aqueous culture medium to assure their viability. The refractive index of cells, $n_{\text {cell }}$ [Fig. 2(a), upper panel] exceeds that of the culture medium, $n_{\text {medium }}(\sim 99 \%$ water) due to presence of $\sim 30 \%$ of organic substances. The imaginary part of the cell refractive index, $\kappa_{\text {cell }}$ [Fig. $2(\mathrm{a})$, lower panel] is determined by the absorption and scattering. The scattering losses dominate in the near-infrared $(\lambda<2.5 \mu \mathrm{m})$ range and the absorption losses dominate in the mid-infrared range $(\lambda>2.5 \mu \mathrm{m})$. 
(a)

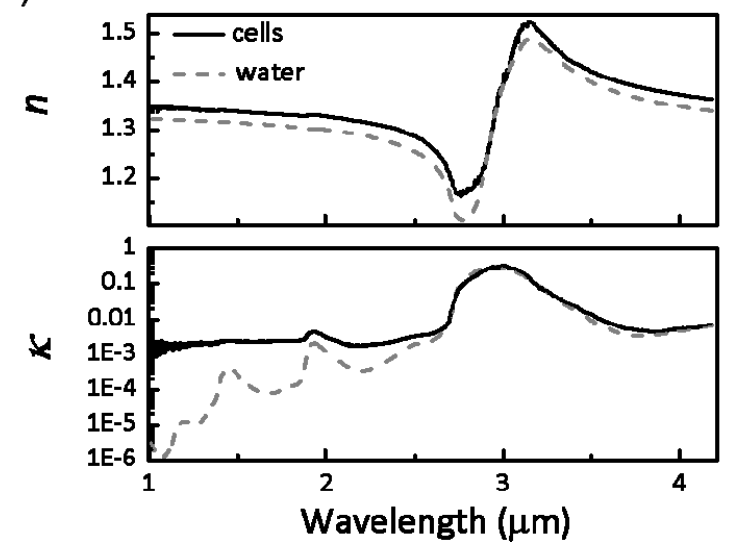

(b)

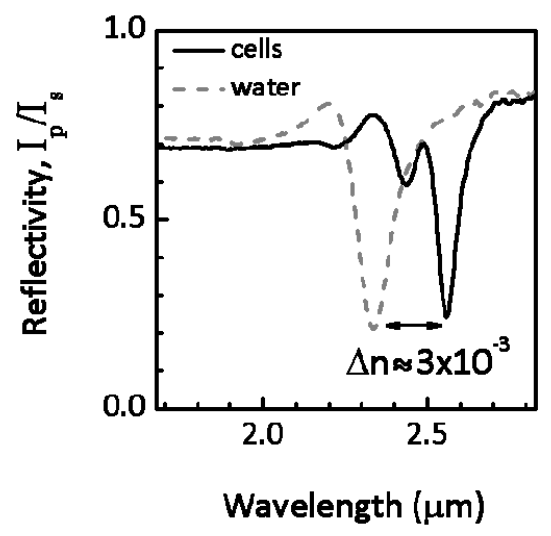

Figure 2. (a) Optical constants of MDCK cells (black solid line) and of water (grey dashed line). Real (upper panel) and imaginary (lower panel) parts of the cell refractive index were inferred from the infrared reflectivity measurements. The optical constants of water were adopted from Bertie et al. ${ }^{8}$. (b) Infrared reflectivity spectra at $\theta_{i n c}=35.1^{0}$ from the MDCK cell monolayer and from water. Both spectra shows sharp dip associated with the surface plasmon resonance. The SP resonant wavelength for cells is shifted towards longer wavelength as the cell refractive index exceeds that of the water.

The analysis of the infrared reflectivity allows following various cellular processes. Figure 2(b) shows infrared reflectivity spectra beyond critical angle, the reflectivity dip corresponding to SP resonance. Position and depth of the resonance are determined by the optical constants $(n, \kappa)$ of the analyte. Any variation in $n$ results in the shift of the resonated wavelength, $\lambda_{S P}$ while variation in $\kappa$ affects mostly the depth of the resonance, $R_{\min }$.

(a)

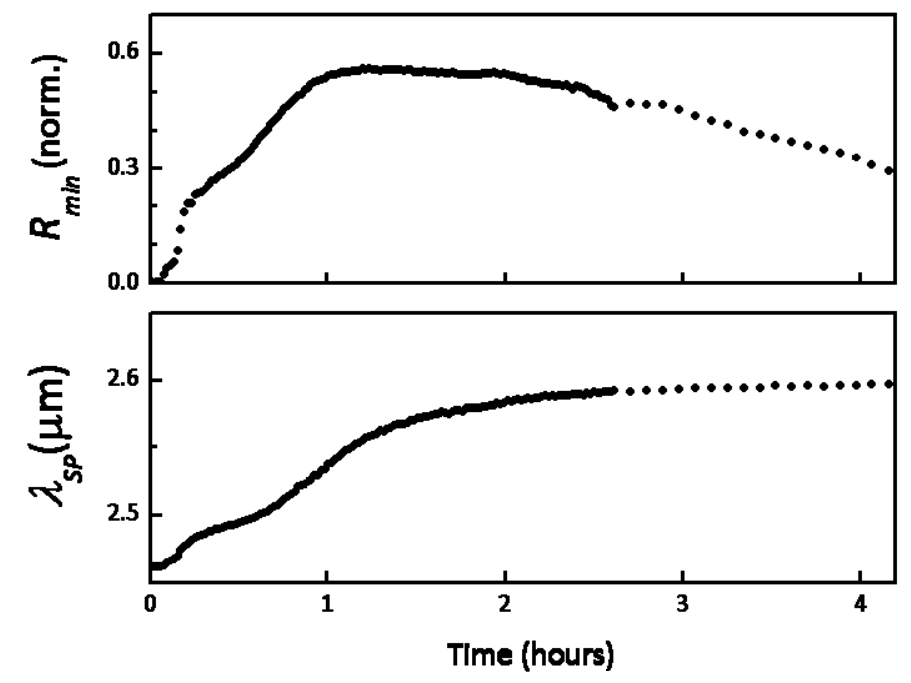

(b)

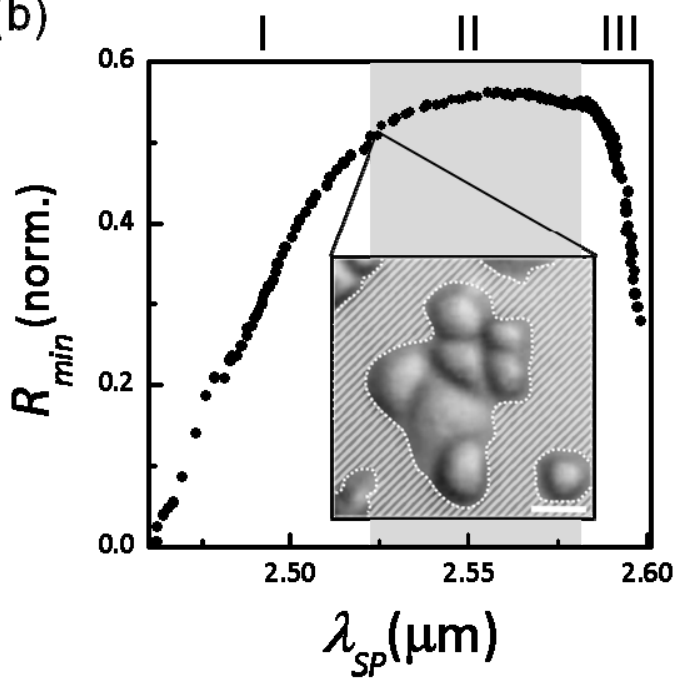

Figure 3. (a) Dynamics of the cell spreading. MDCK cells suspended in MEM were placed on the Au-film ( $\mathrm{t}=0$ ) and allowed to form a continuous cell layer. The upper panel shows the kinetic of SP resonance depth, $R_{\min }$. The lower panel shows the shift of SP wavelength, $\lambda_{S P}$. (b) Dependence of $R_{\min }(\propto P$, the length of the cell-medium interface) on $\Delta n$ ( $\propto f$, cell coverage) reveals distinct stages in cell morphology: I- individual cell spreading; IIcell-cell attachment and formation of cell clusters; III- closure of gaps in contiguous cell sheet. The cell image illustrates the parameters measured by SP. The cell medium interface $(P)$ is denoted by the dashed line, the white stripes show the uncovered Au substrate. The area of these stripes $(1-f)$ allows finding of the cell coverage, $f$.

To demonstrate the potential of the infrared SP for living cell biosensing we present an experiment where MDCK cells form a monolayer on top of the Au-substrate. The shift of $\lambda_{S P}$ allows to follows the change in cell coverage [Fig. 3(a), 
lower panel]. At $\mathrm{t}=0$ MDCK cells suspended in MEM medium were injected into the flow chamber. Cell injection and sedimentation on the Au substrate lasted for $\sim 15 \mathrm{~min}$ and the slow flow $(5 \mu \mathrm{l} / \mathrm{min})$ of fresh media was established later on. The attached cells spread over the substrate and induce the red-shift of $\lambda_{S P}$. The cells form a confluent monolayer after $\sim 2.5$ hours.

The depth of SP resonance $\left(R_{\min }\right)$ tracks the perimeter $(P)$ of cell-medium interfaces at lower (basal) part of the cell [Fig. 3 (b), inset]. Initially, $R_{\min }$ grows as the cells spread on the substrate. Later, it gets smaller as a result of the cell-cell attachment [Fig. 3(a), upper panel]. $R_{\min }\left(\lambda_{S P}\right)$ dependence [Fig. 3(b)] mimics the $P(f)$ dependence. We use this time excluding representation to characterize morphological changes during cell spreading. This plot reveals different stages in cell conformation. Stage I: simultaneous increase of $R_{\min }$ and $\lambda_{S P}$ corresponds to spreading of isolated cells whereas cell area and perimeter grows together. Stage II: the onset of cell-cell attachment, the cell-covered area continues to grow while cells adhere one to another in such a way that the external perimeter does not grow. Stage III: the cell layer closes the remaining gaps that results in the decrease of the external perimeter.

\subsection{Waveguide mode inside intact cells}

Additional spectral feature in reflectivity is specific for the infrared range and results from the waveguide mode excitation inside the interconnected cells. The typical size of cells is of same order of infrared wavelength. In particular MDCK cell monolayer has a typical thickness of $\sim 10 \mu \mathrm{m}$. The progressive decrease of the refractive indices $\left(n_{\text {substrate }}>n_{\text {cell }}>n_{\text {medium }}\right)$ enables the total reflection at the cell-medium interface. On the other hand, the semitransparent Au film acts as a mirror and reflects $\sim 95 \%$ of the incident beam. The strong dispersion of the cell refractive index ( $\left.\mathrm{d} n_{\text {cell }} / \mathrm{d} \lambda\right)$ partially compensates for the non-uniformity of the cell layer thickness $(\sim 1 \mu \mathrm{m})$.

The resonant wavelength of the waveguide modes depends on cell height, $n_{\text {cell }}$, and on the incident angle $\left(\theta_{\text {inc }}\right)$. Based on Fresnel's equations for multilayer structure and known optical constants of each layer we model the reflectivity spectra and extract cell height.

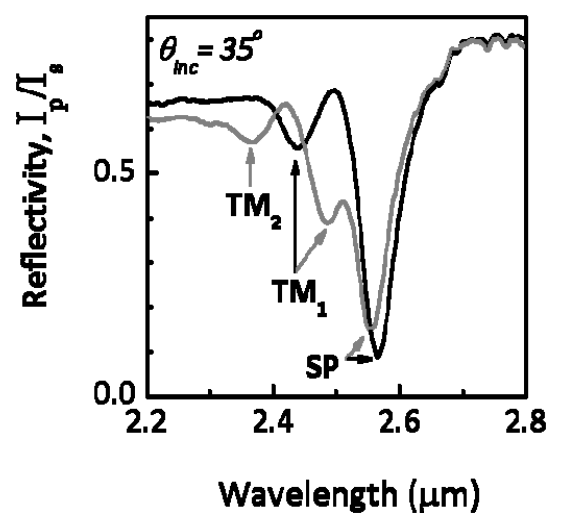

Figure 4. The infrared reflectivity spectra of a MDCK cell monolayer cultured on Au-coated ZnS prism during one day (black line) and during one week (grey line). The resonant wavelength of the waveguide modes $\left(\mathrm{TM}_{1}, \mathrm{TM}_{2}\right)$ resonances reveals the average height of cells $(6.2 \mu \mathrm{m}$ and $9.7 \mu \mathrm{m})$. When the cells are one week old, the cell monolayer is of high quality and we can see the $\mathrm{TM}_{2}$ mode as well. The slight shift of the SP resonance suggests that in this more developed cell layer there is more extracellular medium beneath the cell layer.

Figure 4 illustrates TM modes measured in MDCK cells that were cultured for different time. It should be mentioned that MDCK cells are polarized epithelial cells. The typical time for development of polarized cell phenotype is 2-3 days ${ }^{9}$. During polarization process cells progressively develop columnar morphology. This change in cell morphology is well resolved by the position of $\mathrm{TM}_{1}$ resonance. The cells cultured for longer time has taller shape that indicates on higher degree of cell polarization. It is noteworthy that the SP mode measured on tall cells is blue-shifted. This indicates that along with the cell polarization more extracellular medium enters beneath the cell layer. 


\subsection{Absorption surface-plasmon-based spectroscopy}

Our technique for spectroscopic studies is based on the processing of the infrared reflection spectra in the SP mode and yields the absolute complex refractive index, $n+\mathrm{i} \kappa$ of the examined analyte. We model reflectivity spectra for multilayer assembly using Fresnel equations, modified to account for a specific experimental parameters, such as: (a) incident beam divergence and misalignment; (b) partial polarization of the incident infrared beam; (c) finite extinction ratio of the polarizer; (d) deviation of the optical properties of the Au-film from bulk values.

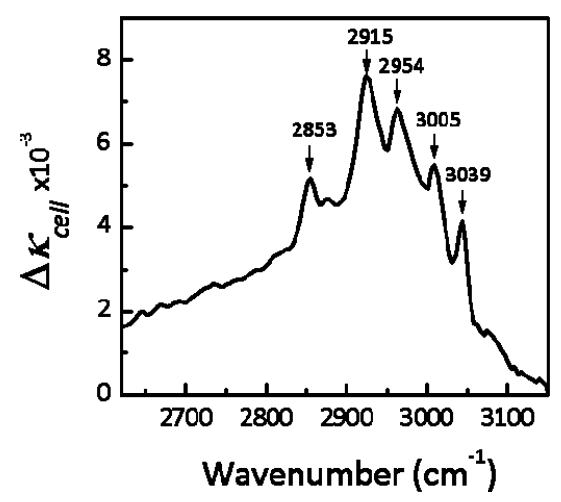

Figure 4. Absorption coefficient of MDCK cells as measured by the infrared SP spectroscopy. The contribution of the organic components of the MDCK cells to, $\Delta \kappa_{\text {cell }}$ in $2700-3150 \mathrm{~cm}^{-1}$ spectral region reveals absorption lines characteristic for $\mathrm{CH}_{\mathrm{n}}$ bonds. The baseline arising from water absorption was subtracted.

The imaginary part of the cell refractive index, $\kappa_{\text {cell }}$ indicates the amount of absorption loss of SP wave. Figure 4(b) shows the scaled absorption spectrum $\left(\Delta \kappa_{\text {cell }}\right)$ for living MDCK cell monolayer at $2400-10000 \mathrm{~cm}^{-1}(1-4.17 \mu \mathrm{m})$ spectral region. To distinguish between absorption contributed by organic compounds (i.e. proteins, lipids and etc.) and water we subtracted $\kappa_{\text {water }}$ baseline from $\kappa_{\text {cell }}: \Delta \kappa_{\text {cell }}=\kappa_{\text {cell }}-A \cdot \kappa_{\text {water }}$, where $\mathrm{A}<1$ is constant. Such correction allows to observe the peaks at $2853,2915,2954,3005$ and $3039 \mathrm{~cm}^{-1}$ which correspond to vibrational levels of $\mathrm{CH}_{\mathrm{n}}$ (aliphatic) bonds characteristic to lipids ${ }^{10-13}$.

\section{SUMMARY}

We demonstrated here the label-free biosensing based on surface plasmon infrared reflection spectroscopy. This method is highly sensitive to changes in refractive index, not less than the visible surface plasmon sensors. However, in infrared range it has several features unique for whole cell sensing. The infrared SP is sensitive to changes taking place up to several microns away from the cell- metal interface. The SP resonance provides information not only about the total biomass but cell morphology as well. We showed how simultaneous analysis of the SP shift and depth probes lateral organization of the cells on the substrate and distinguishes between different stages in cell spreading.

The waveguide modes traveling in cell monolayer are another feature specific for the infrared range. Analysis of these modes yields the height and integrity of the cell monolayer. These properties have been conventionally measured with confocal fluorescent microscopy which has limited time and space resolution and demands fluorescent probes ${ }^{14}$.

Spectroscopy of living cells in the SP regime yields absorbance spectra of aqueous samples, such living cells. The main advantage of the SP based infrared spectroscopy is the field enhancement that allows using single reflection as opposed to multiple reflections used in attenuated total reflection infrared spectroscopy (ATR). In addition, SP evanescent wave penetrates much deeper into the sample compared with conventional evanescent wave in the ATR regime ${ }^{15}$.

Our current development provides multiparameter sensing of living cells and can serve as a powerful experimental strategy for cell studies in health and disease.

\section{ACKNOWLEDGMENTS}

We are grateful to Dr. Efrat Zlotkin-Rivkin and Leorah Kharilker for assistance in cell culture and Dr. Boaz Ran and Dr. Tsafrir Bravman (Bio-Rad, Haifa, Israel) for constant encouragement and stimulating discussion. This work was 
supported by the NOFAR grant of the Israel Ministry of Industry and Trade. We are grateful the Yissum Research \& Development Company of the Hebrew University of Jerusalem for financial support. V. L. acknowledges support from the Lady Davis Fellowship Foundation.

\section{REFERENCES}

[1] M. Golosovsky, V. Lirtsman, V. Yashunsky et al., "Midinfrared surface-plasmon resonance: A novel biophysical tool for studying living cells," Journal of Applied Physics, 105(10), 1020-1021 (2009).

[2] V. Lirtsman, M. Golosovsky, and D. Davidov, "Infrared surface plasmon resonance technique for biological studies," Journal of Applied Physics, 103(1), 014702 (2008).

[3] V. Yashunsky, S. Shimron, V. Lirtsman et al., "Real time monitoring of epithelial cell-cell and cell-substrate interactions by infrared surface plasmon spectroscopy," Submitted to Biophysical Journal, (2010).

[4] V. Yashunsky, S. Shimron, V. Lirtsman et al., "Real-Time Monitoring of Transferrin-Induced Endocytic Vesicle Formation by Mid-Infrared Surface Plasmon Resonance," Biophysical Journal, 97(4), 1003-1012 (2009).

[5] H. Raether, "Surface-Plasmons on Smooth and Rough Surfaces and on Gratings," Springer Tracts in Modern Physics, 111, 1-133 (1988).

[6] K. Johansen, H. Arwin, I. Lundstrom et al., "Imaging surface plasmon resonance sensor based on multiple wavelengths: Sensitivity considerations," Review of Scientific Instruments, 71(9), 3530-3538 (2000).

[7] W. Knoll, "Interfaces and thin films as seen by bound electromagnetic waves," Annual Review of Physical Chemistry, 49, 569-638 (1998).

[8] J. E. Bertie, and Z. D. Lan, "Infrared intensities of liquids .20. The intensity of the OH stretching band of liquid water revisited, and the best current values of the optical constants of $\mathrm{H} 2 \mathrm{O}(1)$ at 25 degrees $\mathrm{C}$ between 15,000 and 1 cm(-1)," Applied Spectroscopy, 50(8), 1047-1057 (1996).

[9] E. Rodriguez-Boulan, and W. J. Nelson, "Morphogenesis of the polarized epithelial cell phenotype," Science, 245(4919), 718-25 (1989).

[10]F. Draux, P. Jeannesson, C. Gobinet et al., "IR spectroscopy reveals effect of non-cytotoxic doses of anti-tumour drug on cancer cells," Analytical and Bioanalytical Chemistry, 395(7), 2293-2301 (2009).

[11] P. Lasch, M. Boese, A. Pacifico et al., "FT-IR spectroscopic investigations of single cells on the subcellular level," Vibrational Spectroscopy, 28(1), 147-157 (2002).

[12] S. M. LeVine, J. D. Radel, J. A. Sweat et al., "Microchemical analysis of retina layers in pigmented and albino rats by Fourier transform infrared microspectroscopy," Biochimica Et Biophysica Acta-General Subjects, 1473(2-3), 409-417 (1999).

[13] L. M. Miller, and P. Dumas, "Chemical imaging of biological tissue with synchrotron infrared light," Biochimica Et Biophysica Acta-Biomembranes, 1758(7), 846-857 (2006).

[14]D. Axelrod, and G. M. Omann, “Combinatorial microscopy,” Nature Reviews Molecular Cell Biology, 7(12), 944952 (2006).

[15] M. K. Kuimova, K. L. A. Chan, and S. G. Kazarian, "Chemical Imaging of Live Cancer Cells in the Natural Aqueous Environment," Applied Spectroscopy, 63(2), 164-171 (2009). 